\title{
Contents
}

1 An Overview of the Solar-Terrestrial Environment

A.C.-L. Chian, Y. Kamide
$1.1 \quad$ Introduction ................................... 2

1.2 Overview and History of Solar-Terrestrial Environment Research ..... 2

1.2 .1 Sun....................................... 2

1.2.2 Solar Wind ..................................... 5

1.2.3 Magnetosphere-lonosphere-Thermosphere............... 7

1.2.4 Geomagnetism and Geomagnetic Storms/Substorms ............ 9

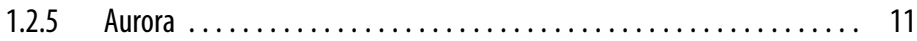

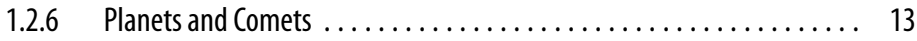

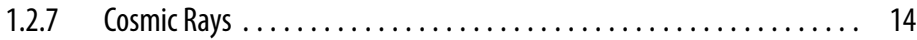

1.3 Nature of the Solar-Terrestrial Environment $\ldots \ldots \ldots \ldots \ldots \ldots \ldots . \ldots \ldots$

1.3.1 Linear Waves ..................................... 15

1.3 .2 Instabilities ................................... 16

1.3.3 Nonlinear Waves ..................................... 17

1.3 .4 Turbulence ......................................... 18

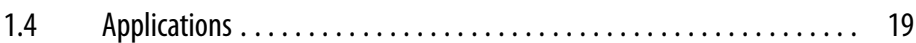

1.4.1 Space Weather and Space Climate $\ldots \ldots \ldots \ldots \ldots \ldots \ldots \ldots \ldots \ldots \ldots$

1.4.2 Plasma Astrophysics ............................... 20

1.4.3 Controlled Thermonuclear Fusion .......................... 21

$1.5 \quad$ Concluding Remarks............................. 21

References .................................... 22

\section{Part 1 The Sun}

2 The Solar Interior - Radial Structure, Rotation, Solar Activity Cycle

A. Brandenburg
$2.1 \quad$ Introduction $\ldots \ldots \ldots \ldots \ldots \ldots \ldots \ldots \ldots \ldots \ldots \ldots \ldots, 28$

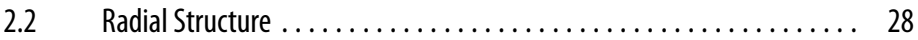

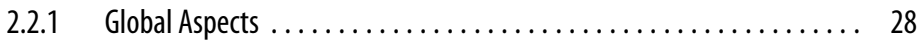

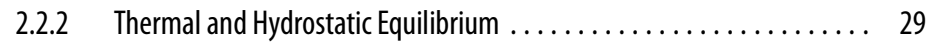

2.2.3 Transition to Adiabatic Stratification $\ldots \ldots \ldots \ldots \ldots \ldots \ldots \ldots . \ldots \ldots$

2.2.4 Mixing Length Theory and Convection Simulations .............. . 31

2.3 Helioseismology $\ldots \ldots \ldots \ldots \ldots \ldots \ldots \ldots \ldots \ldots \ldots \ldots \ldots$

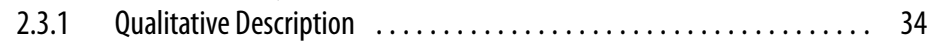

2.3.2 Inverting the Frequency Spectrum $\ldots \ldots \ldots \ldots \ldots \ldots \ldots \ldots \ldots$

2.3.3 The Solar Abundance Problem . . . . . . . . . . . . . . . . . . . . . . 37

2.3.4 Internal Solar Rotation Rate ......................... 37

2.3.5 Local Helioseismology $\ldots \ldots \ldots \ldots \ldots \ldots \ldots \ldots \ldots \ldots \ldots \ldots$ 
$2.4 \quad$ Solar Activity Cycle $\ldots \ldots \ldots \ldots \ldots \ldots \ldots \ldots \ldots \ldots \ldots \ldots \ldots$

2.4.1 The Butterfly Diagram ......................... 38

2.4.2 Cyclic Activity on Other Solar-Like Stars ............... 39

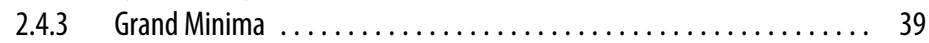

2.4.4 Active Regions and Active Longitudes ................. 39

2.4 .5 Torsional Oscillations .......................... 40

2.5 Dynamo Theory ............................. 41

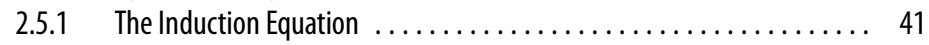

2.5.2 Small Scale Dynamo Action $\ldots \ldots \ldots \ldots \ldots \ldots \ldots \ldots \ldots \ldots \ldots .42$

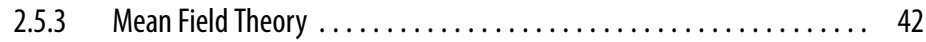

2.5.4 Numerical Determination of $\alpha \ldots \ldots \ldots \ldots \ldots \ldots \ldots \ldots \ldots \ldots$

2.5.5 0ther Effects................................. 44

2.6 Models of the Solar Cycle ........................... 45

2.6.1 One-Dimensional Models ........................... 45

2.6.2 Different Solar Dynamo Scenarios ..................... 45

2.6 .3 Nonlinear Saturation $\ldots \ldots \ldots \ldots \ldots \ldots \ldots \ldots \ldots \ldots \ldots . \ldots \ldots$

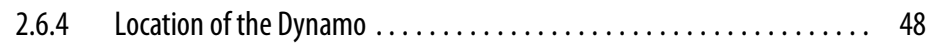

2.7 Differential Rotation............................... 48

2.7.1 Mean Field Theory of Differential Rotation ................ 48

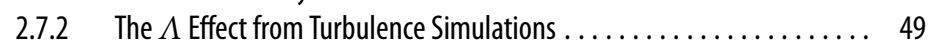

2.7.3 Meridional Flow and the Baroclinic Term . . . . . . . . . . . 50

2.7.4 Near-Surface Shear Layer . . . . . . . . . . . . . . . . . . . . . 51

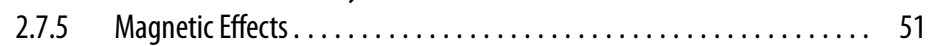

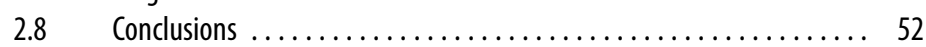

References $\ldots \ldots \ldots \ldots \ldots \ldots \ldots \ldots \ldots \ldots \ldots \ldots \ldots \ldots \ldots \ldots$

3 Solar Atmosphere

$3.1 \quad$ Introduction $\ldots \ldots \ldots \ldots \ldots \ldots \ldots \ldots \ldots \ldots \ldots \ldots \ldots \ldots, 56$ E.R. Priest

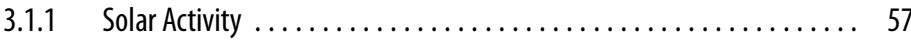

3.1.2 The Solar Revolution $\ldots \ldots \ldots \ldots \ldots \ldots \ldots \ldots \ldots \ldots \ldots \ldots$

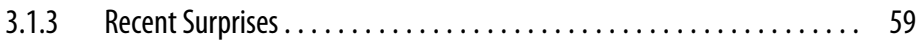

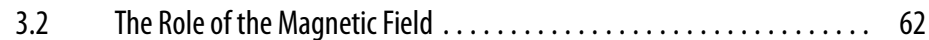

3.2 .1 Basic Equations ................................. 62

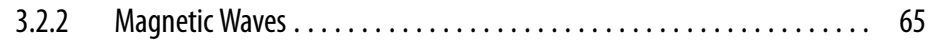

3.2 .3 Magnetic Reconnection ........................ 66

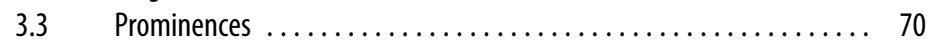

$3.4 \quad$ Solar Flares .................................. 71

3.4.1 Introduction ................................ 71

3.4.2 Energy Release by Magnetic Reconnection . . . . . . . . . . . . . . 73

3.4.3 Conditions for Flare 0 ccurrence $\ldots \ldots \ldots \ldots \ldots \ldots \ldots \ldots \ldots \ldots$

3.4.4 Catastrophe and Instability Models for Eruption ............. 76

$3.5 \quad$ Coronal Heating .............................. 76

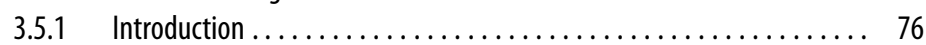

3.5.2 Numerical Experiment on Global Active Region Heating . . . . . . . . 80

3.5 .3 Heating by MHD Waves $\ldots \ldots \ldots \ldots \ldots \ldots \ldots \ldots \ldots \ldots \ldots . \ldots \ldots$

3.5.4 Heating by Magnetic Reconnection $\ldots \ldots \ldots \ldots \ldots \ldots \ldots \ldots \ldots 81$

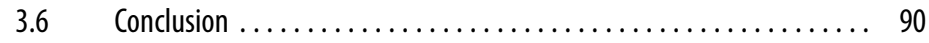

References $\ldots \ldots \ldots \ldots \ldots \ldots \ldots \ldots \ldots \ldots \ldots \ldots \ldots \ldots$ 
4 Solar Wind

E.N. Parker
5 Coronal Mass Ejection P.J. Cargill and L.K. Harra

6 Solar Radio Emissions J.-L. Bougeret and M. Pick

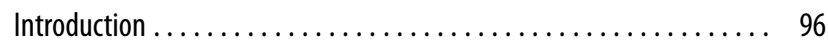

The Corona .................................. 96

Outward Decline of Density and Pressure . . . . . . . . . . . . . . . 97

Comets and Solar Corpuscular Radiation .................. 98

Cosmic Ray Variations . . . . . . . . . . . . . . . . . . . . ....... 98

Plasma in Interplanetary Space $\ldots \ldots \ldots \ldots \ldots \ldots \ldots \ldots \ldots . \ldots \ldots$

The State of the Corona .......................... 100

Theoretical Foundations of Hydrodynamics and Magnetohydrodynamics 101

Kinetic Conditions in the Corona ..................... 103

Magnetohydrodynamics........................ 104

Hydrodynamic Expansion of the Solar Corona . . . . . . . . . . . . . . 105

Sufficient Conditions on Coronal Temperature .............. 107

Analogy with Expansion Through a Laval Nozzle .............. 108

Gravitational Throttling of Coronal Expansion.................... 109

Wind Density and Solar Mass Loss . . . . . . . . . . . . . . . . . . 110

Magnetic Fields and Streams in the Solar Wind ............ 112

Discussion ................................. 113

References ................................... 114

$5.1 \quad$ Introduction $\ldots \ldots \ldots \ldots \ldots \ldots \ldots \ldots \ldots \ldots \ldots \ldots \ldots \ldots \ldots \ldots$

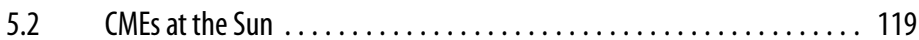

5.2 .1 Properties ................................. 120

5.2.2 What Causes CMEs: Observational Evidence .................. 121

5.2 .3 Theoretical Ideas ............................. 125

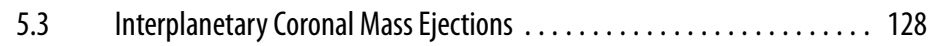

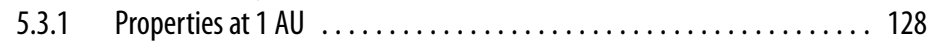

5.3.2 Putting the Solar and Interplanetary Parts Together........... 130

5.4 Conclusions and Future Prospects .................. 130

References ............................ 131

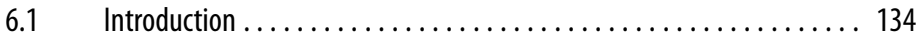

6.2 Radio Wave Propagation ....................... 135

$6.2 .1 \quad$ Basics ...................................... 135

6.2 .2 Scattering of Radio Waves ....................... 137

6.3 Thermal Radiation from the Sun ....................... 137

6.3.1 Microwave Domain . . . . . . . . . . . . . . . . . . . . . . . . . . . . . . 137

6.3.2 Decimeter-Meter Domain .............................. 137

$6.4 \quad$ Solar Radio Bursts ............................... 138

6.4.1 Emission Mechanisms .......................... 139

6.4.2 Electron Beams ............................... 139

6.4.3 Remote Tracking of Collisionless Shock Waves ................ 141

6.4.4 Radio Emission Following Flares and Large-Scale Disturbances . . . . . . 142

$6.5 \quad$ In Situ Wave and Particle Measurements ................ 144

6.6 Radio Signatures of Coronal and Interplanetary Coronal Mass Ejections . 145

6.6.1 Flare/CME Events: Lift-Off and Angular Spread in the Corona . . . . . . . 146

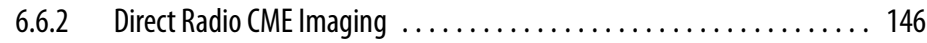

6.6.3 Interplanetary Coronal Mass Ejections . ................. 147 
6.7 Conclusions: The Relevance of Radio Observations to the Understanding of the Solar-Terrestrial Environment ....................... 148

References ...................................... 150

\section{Part 2 The Earth}

7 Magnetosphere

M. Schulz

8 lonosphere

P.-L. Blelly and D. Alcaydé

9 Thermosphere

S. Kato

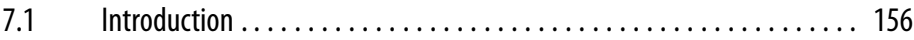

7.2 Magnetic Configuration ............................. 157

7.3 Magnetospheric Electric Fields ........................... 164

7.4 Magnetospheric Charged Particles ......................... 173

7.5 Summary ..................................... 186

References ........................................ 187

$8.1 \quad$ Production and Structure . . . . . . . . . . . . . . . . . . . . . . . . . . . 190

$8.1 .1 \quad$ Ionization Processes . . . . . . . . . . . . . . . . . . . . . . . . . . . . . . . . . 190

8.1.2 Primary lonospheric Outputs .............................. 194

8.1 .3 Ionospheric Structure ................................. 196

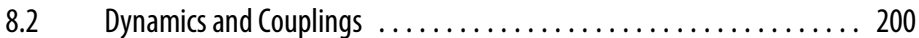

8.2 .1 Electrodynamics.................................. 200

8.2.2 Energetics ...................................... 204

8.2 .3 Field Aligned Transport $\ldots \ldots \ldots \ldots \ldots \ldots \ldots \ldots \ldots \ldots \ldots \ldots \ldots \ldots \ldots$

$8.2 .4 \quad$ Coupling Processes ................................ 212

$8.3 \quad$ Observations and Modeling . . . . . . . . . . . . . . . . . . . . . . . 214

8.3 .1 Incoherent Scatter . . . . . . . . . . . . . . . . . . . . . . . . . . . . . 214

8.3.2 Coherent Radars ................................. 215

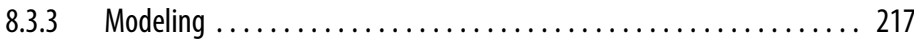

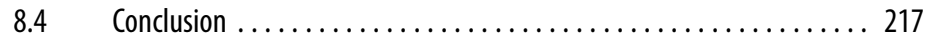

References ....................................... 219

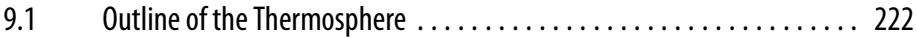

9.2 Basic Thermosphere Dynamics .......................... 224

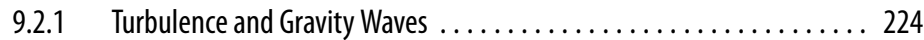

9.2.2 Waves and Winds ..................................... 229

$9.3 \quad$ GCM Simulation ..................................... 234

9.4 Observation ....................................... 236

9.5 Dynamics of the Polar Thermosphere ....................... 239

$9.6 \quad$ Concluding Remarks................................ 242

References .................................... 242 


\section{Part 3 Space Plasmas}

10 Space Plasmas

C. Uberoi

11 Magnetic Reconnection A. Nishida

12 Nonlinear Plasmas

L. Stenflo and P.K. Shukla
10.1 Characteristic Properties of Plasmas .................... 250

10.2 Particles in Space Plasmas . . . . . . . . . . . . . . . . . . . . . . . . . 252

10.2.1 Motion of Charged Particles in a Uniform Magnetic Field . . . . . . . . . 252

10.2.2 Particle Drifts .............................. 253

10.2.3 Magnetic Mirrors ............................. 255

10.2.4 Motion in a Dipole Magnetic Field $\ldots \ldots \ldots \ldots \ldots \ldots \ldots \ldots \ldots 256$

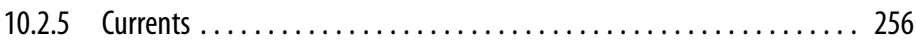

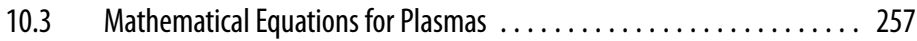

10.4 Plasma as an MHD Fluid . . . . . . . . . . . . . . . . . . . 259

10.4.1 MHD Equations................................. 260

10.4.2 Motion of the Magnetic Field . . . . . . . . . . . . . . . . . . . . . 261

10.4 .3 Hydromagnetic Equilibrium ..................... 263

10.4.4 Transport Coefficients: Electrical Conductivity .............. 263

10.5 Waves in Space Plasmas . . . . . . . . . . . . . . . . . . . . . . . . . 264

10.5.1 Electromagnetic Waves ............................ 264

10.5.2 Dielectric Constant for Magnetized Plasma . . . . . . . . . . . . . . . . . 266

10.5 .3 Electrostatic Waves. . . . . . . . . . . . . . . . . . . . . . . . 268

10.5.4 Magnetohydrodynamic Waves .......................... 269

10.5.5 Kinetic Theory of Plasma Waves ....................... 270

10.5.6 Surface Waves $\ldots \ldots \ldots \ldots \ldots \ldots \ldots \ldots \ldots \ldots \ldots \ldots \ldots \ldots \ldots$

10.6 Equilibria and Their Stability $\ldots \ldots \ldots \ldots \ldots \ldots \ldots \ldots \ldots \ldots \ldots \ldots \ldots \ldots$

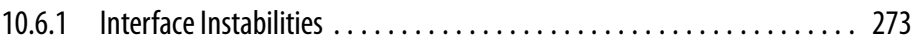

10.6 .2 Two-stream Instability . . . . . . . . . . . . . . . . . . . . 275

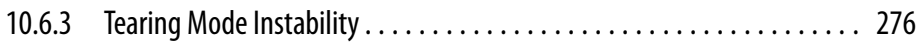

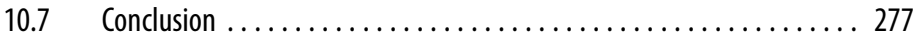

References ............................. 277

11.1 Introduction . . . . . . . . . . . . . . . . . . . . . . . . . . 280

11.2 Reconnection on the Magnetopause $\ldots \ldots \ldots \ldots \ldots \ldots \ldots \ldots . \ldots 281$

11.2.1 Formation and Topology of Open Field Lines .............. 281

11.2.2 Structure of the Magnetopause in Terms of Magnetohydrodynamics ... 283

11.2.3 Direct Consequences of the Magnetopause Reconnection ......... 289

11.3 Reconnection Inside the Magnetotail . . . . . . . . . . . . . . . . . . . 292

11.3.1 Sites of the Magnetotail Reconnection . . . . . . . . . . . . . . . . . . . . . 292

11.3.2 Structure of the Reconnection Region . . . . . . . . . . . . . . . . . . 294

11.3.3 Consequences of the Magnetotail Reconnection ............... 301

11.3.4 Cause of the Near-Earth Reconnection ................... 306

References ............................... 308

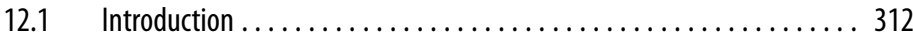

12.2 Stimulated Scattering of Electromagnetic Waves . . . . . . . . . . . . . . 312

12.3 Resonant Three-Wave Interactions in Plasmas . . . . . . . . . . . 316

12.4 Parametric Instabilities of Magnetic Field-Aligned Alfvén Waves . . . . 318

12.5 Kinetic Alfvén Waves Driven Zonal Flows ............... 320 


\section{Part 4 Processes in the Solar-Terrestrial Environment}

13 The Aurora

B. Hultqvist

14 Geomagnetic Storms Y. Kamide and Y.P. Maltsev

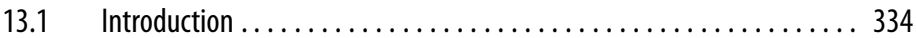

13.2 Geographical Distributions ........................... 334

13.3 Spectrum, Optical Intensity and Power . . . . . . . . . . . . . . . . . 337

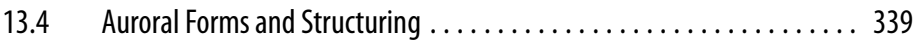

13.5 Auroral Substorms and Storms ........................... 342

13.6 Auroral Electrodynamics and Energetic Particle Precipitation ........ 344

13.7 Correlations of Aurora with Various Solar and Geophysical Phenomena . 348

$13.8 \quad$ Aurora as a Source of Plasma ............................ 349

13.9 The Aurora as a Universal Phenomenon . . . . . . . . . . . . . . . . . . . 351

13.10 Concluding Remarks............................... 352

References .................................... 352

$14.1 \quad$ Introduction .................................... 356

14.2 What is a Geomagnetic Storm? ..................... 356

14.3 Ring Current as a Dominant Signature of Geomagnetic Storms........ 358

14.4 Solar Wind Causes of Geomagnetic Storms ................... 359

14.5 Magnetospheric Geometry During Geomagnetic Storms .......... 362

14.5 .1 Auroral Electrojets . . . . . . . . . . . . . . . . . . . . . . . . . . . . 362

14.5.2 Auroral Oval .................................... 362

14.5.3 Standoff Distance ..................................... 363

14.5.4 Stable Trapping Boundary ......................... 363

14.6 Storm-Time Magnetic Fields and Electric Fields in the Magnetosphere .. 363

14.6.1 Spatial Distribution of the Electric Currents. . . . . . . . . . . . . . . 363

14.6.2 Contribution of Different Current Systems to Dst . . . . . . . . . . . . . . . 364

14.6.3 Storm-Time Electric Fields in the Magnetosphere . . . . . . . . . . . . . . 366

14.7 Discussion on Open Issues of Geomagnetic Storm Dynamics ......... 367

14.7.1 Influence of the Solar Wind Parameters on Dst .................... 367

14.7.2 Influence of the Substorm Expansion Phase on Dst .............. 368

14.7.3 What Causes Storm-Time Equatorward Shift of the Auroral Oval? . . . . . 370

14.7.4 Why Does the IMF Southward Component Affect Dst? . . . . . . . . . 371

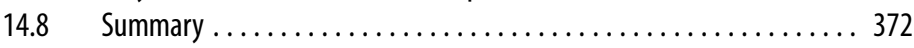

References ..................................... 373 
15 Substorms G. Rostoker

16 Ultra Low Frequency Waves in the Magnetosphere

U. Villante
$15.1 \quad$ Observations .......................................... 376

15.1.1 Historical Perspective . .............................. 376

15.1.2 Observational Basis for the Evolution of the Substorm Concept: Pre-Satellite Era . . ................................ 377

15.1.3 Observational Basis for the Evolution of the Substorm Concept: Satellite Era ................................. 378

15.1.4 The Solar Wind as a Driver of Substorm Activity ............... 381

15.1.5 Evolution of a Substorm Optically and Magnetically: a Case Study .... 382

15.2 Physical Frameworks for Understanding Substorms ............. 386

15.2.1 The Near-Earth Neutral Line (NENL) Framework . . . . . . . . . . . . 386

15.2.2 Near-Earth Current Disruption Framework . . . . . . . . . . . . . . . . 388

15.2.3 Boundary Layer Dynamics Model . . . . . . . . . . . . . . . . . . . . . . . 389

15.3 Final Comments ................................... 391

References ................................... 393

$16.1 \quad$ Introduction ....................................... 398

16.2 Linear Theory of Hydromagnetic Waves . . . . . . . . . . . . . . . 400

16.2.1 The Uniform Field . . . . . . . . . . . . . . . . . . . . . . . . . . . . . . . . 400

16.2.2 The Dipole Field ..................................... 401

16.3 Sources of Geomagnetic Pulsations .................... 402

16.3.1 Upstream Waves ................................ 402

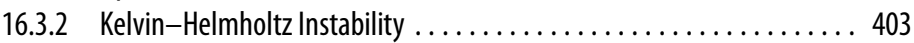

16.3 .3 Ion-Cyclotron Instability .......................... 403

16.4 Effects of the lonosphere and Field Line Eigenperiods ........... 404

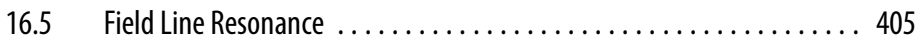

16.6 Cavity Resonance ................................. 407

16.7 Low Frequency Pulsations . . . . . . . . . . . . . . . . . . . . 407

16.8 Mid-Frequency Pulsations . .......................... 409

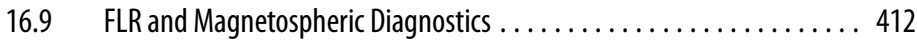

16.10 Cavity/Waveguide Modes ............................. 414

16.11 High Frequency Pulsations . . . . . . . . . . . . . . . . . . . . . . . . . . 415

16.12 Irregular Pulsations ............................. 417

16.13 Concluding Remarks............................ 419

References ................................... 419

$17.1 \quad$ Introduction .................................... 424

17.2 Early Technologies: Telegraph and Wireless ................. 425

17.3 Growth in Electrical Technologies ...................... 427

17.4 The Space Age and Space Weather . . . . . . . . . . . . . . . . . . . . 429

17.4.1 Ionosphere and Earth Currents . . . . . . . . . . . . . . . . . . . . . . 429

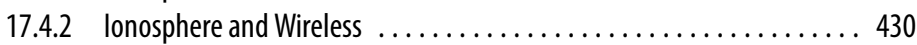

17.4.3 Solar Radio Noise ....................................... . 431

17.4.4 Space Radiation Effects $\ldots \ldots \ldots \ldots \ldots \ldots \ldots \ldots \ldots \ldots \ldots . \ldots . \ldots \ldots$

17.4.5 Magnetic Field Variations ............................ 436

17.4.6 Micrometeoroids (and Space Debris) . .................. 436

17.4.7 Atmosphere: Low Altitude Spacecraft Drag . . .............. 436

17.4.8 Atmosphere Water Vapor ........................... 437 
18 Effects of the Solar Cycle on the Earth's Atmosphere K. Labitzke

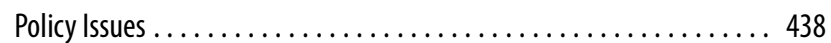

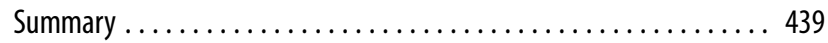

References ..................................... 439

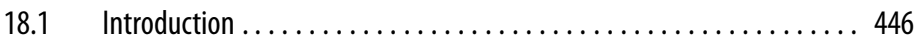

18.2 Data and Methods ............................ 446

18.3 Variability in the Stratosphere $\ldots \ldots \ldots \ldots \ldots \ldots \ldots \ldots \ldots . \ldots 44$

18.4 Influences of the 11-Year Sunspot Cycle on the Stratosphere . . . . . . . 448

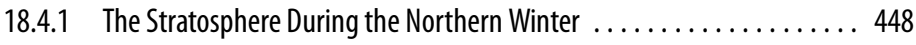

18.4.2 The Stratosphere During the Northern Summer . . . . . . . . . . . 453

18.5 The Solar Signal in the Troposphere $\ldots \ldots \ldots \ldots \ldots \ldots \ldots \ldots . \ldots 458$

18.6 The QB0-Solar-Relationship Throughout the Year ............. 459

18.7 Models and Mechanisms ........................... 461

18.8 Summary .................................. 463

References ................................. 464

\section{Part 5 Planets and Comets in the Solar System}

\section{Planetary Magnetospheres}

M.G. Kivelson

20 The Solar-Comet Interactions

D.A. Mendis
19.1 Introduction .................................. 470

19.2 Parameters that Control Magnetospheric Configuration and Dynamics . 470

19.2.1 Properties of the Flowing Plasma . . . . . . . . . . . . . . . . 470

19.2.2 Properties of the Planet or Moon . .................. 473

19.2.3 Dimensionless Ratios Controlling Size and Dynamics . . . . . . . . . . 473

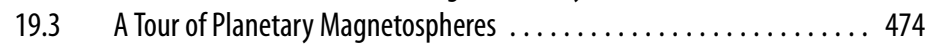

19.3.1 Mini-Magnetospheres ....................... 474

19.3.2 Giant Magnetospheres of Rapidly Rotating Planets ........... 480

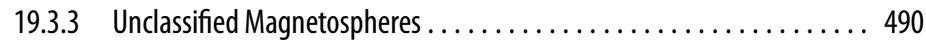

19.3.4 Mars: a Special Case . . . . . . . . . . . . . . . . . . . . . . . 490

19.4 Summary: some Lessons for Earth $\ldots \ldots \ldots \ldots \ldots \ldots \ldots \ldots \ldots . \ldots 49$

References ................................ 492

$20.1 \quad$ Introduction ................................ 494

20.2 Cometary Reservoirs................................. 494

20.3 The Nature of the Cometary Nucleus . . . . . . . . . . . . . . . . 496

20.4 Interaction with Solar Radiation ...................... 498

20.5 The Interaction with the Solar Wind . . . . . . . . . . . . . . . . . 501

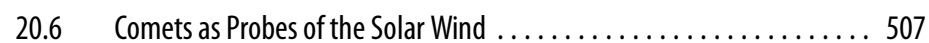

References ................................ 514

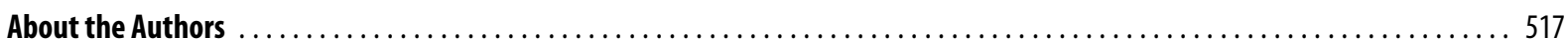

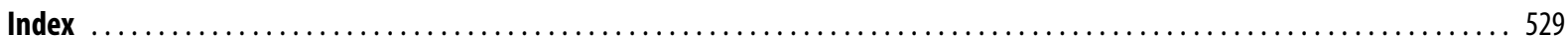

